\title{
A Novel Timing Synchronization Algorithm based on Signal Reconstruction in Multipath Fading Channel
}

\author{
Juan CHEN, Xueqiang ZHENG* \\ Institute of Communications Engineering \\ PLA University of Science and Technology \\ Nanjing, Jiangsu, China \\ *E-mail: pumpkin0910@163.com \\ E-mail: zxqq302@163.com \\ *Corresponding author
}

\begin{abstract}
Timing synchronization method for DS-SS system taking into account the multipath effect on HF channel is studied. A novel timing synchronization algorithm based on signal reconstruction is proposed in multipath fading HF channel. Through signal reconstructing and interference cancelling, the right path position and multipath delay can be evaluated. The simulation results show that the detection performance can be improved, and this new timing algorithm can easily separate different paths which cannot be done by traditional RAKE receiver.
\end{abstract}

Keywords-Synchronization; Signal Reconstructing; Multipath delay; HF channel

\section{INTRODUCTION}

Direct-sequence spread-spectrum (DS-SS) techniques have found extensive applications in modern communication systems such mobile communications, satellite communications and HF communications [1-3]. To exploit the excellent advantages of DS-SS techniques, the receiver must be capable of synchronizing a locally generated pseudonoise (PN) code with an incoming PN sequence. There are two steps of synchronization, which are acquisition and tracking [4-7]. We focus on the acquisition step of DS-SS signals in HF channels.

The HF channel is a time-varying fading channel, involving time delay, Doppler frequency shift, interference and noise [8]. The typical multipath delay are $2 \sim 5 \mathrm{~ms}$. Under the influence of fading and multipath delay, the signal recognition and synchronization cannot be done easily. The typical method to solve the problem is RAKE receiver which can combine the multipath signal to negative the influence of fading [9-11]. However, this method can only work well under the situation which the multipath can be separated obviously.

In this paper, we address some further work to against multipath and fading influence on synchronization. A novel timing synchronization algorithm based on signal reconstruction is proposed in multipath fading HF channel. Through signal reconstructing and interference cancelling, the right path situation and multipath delay can be evaluated. The simulation results show that the detection performance can be improved, and this new timing algorithm can easily separate different paths which cannot be done by traditional RAKE receiver.

The paper is organized as follows. In Section II, the system model is briefly introduced. In Section III, the signal reconstruction method is given. In Section IV, the novel timing synchronization algorithm based on signal reconstruction is proposed in multipath fading $\mathrm{HF}$ channel is given. In Section $\mathrm{V}$, the simulation results are shown. Finally, the conclusion is given in Section VI.

\section{SYSTEM MODEL}

The sampled received signal has two hypotheses. Hypothesis $H_{1}$ denotes the receiving signal is active, and hypothesis $H_{0}$ denotes the receiving signal is inactive.

$$
\begin{aligned}
& H_{1}: \tilde{r}(i)=\sum_{k=1}^{\mathrm{N}} h_{k} * s(i)+n(i) \\
& H_{0}: \tilde{r}(i)=n(i)
\end{aligned}
$$

where $r(i)$ is the signal received by the receiver, ${ }^{s(i)}$ is the transmitted signal, $\mathrm{N}$ is the number of the multipath. The signal $s(i)$ is distorted by the channel gain $h_{\mathrm{k}}$, which is assumed to be constant during the detection interval, and is further corrupted by the zero-mean additive white Gaussian noise $^{n(i)}$ with the variance $\sigma_{n}^{2}$. Without loss of generality, $s(i)$ and $n(i)$ are assumed to be independent of each other. $s n r_{k}=\left|h_{k}\right|^{2} E_{s} / \sigma^{2}$ denotes the signal-to-noise ratio (SNR), where $E_{s}$ is the signal energy.

The typical DS-SS synchronization model is illustrated in figure 1 . 


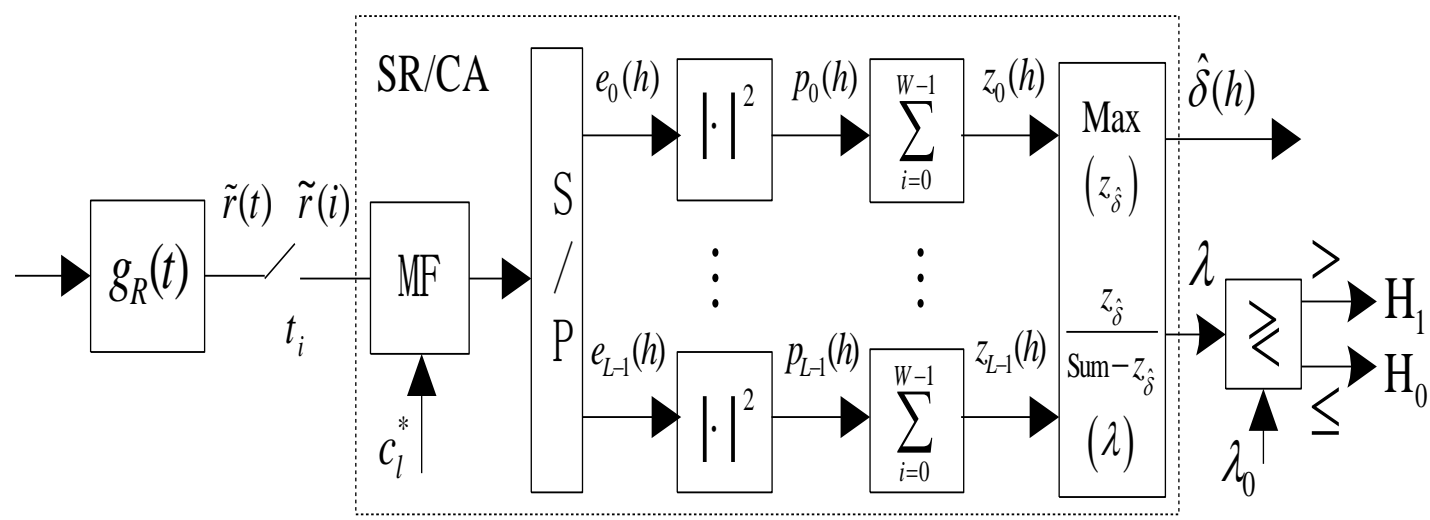

Figure 1. Synchronization model

The output of the local PN code $c=\left[c_{0}, \ldots c_{l}, \ldots c_{L-1}\right]$ match filter is

$$
\begin{aligned}
& e_{l}(h)=\sum_{i=0}^{L-1} c_{i}^{*} \tilde{r}(h L+l+i) \\
& p_{l}(h)=\left|e_{l}(h)\right|^{2} \\
& z_{l}(h)=\sum_{i=0}^{W-1} p_{l}(h-i)
\end{aligned}
$$

where $\mathrm{L}$ is length of $\mathrm{PN}$ code, $\mathrm{W}$ is the smoothing factor.

We define these variables :

$$
\begin{gathered}
z_{\max }(h) \square \max \left\{z_{0}(h), z_{1}(h), \cdots, z_{L-1}(h)\right\} \\
\left.\hat{\delta}(h)\right|_{z_{\hat{\delta}(h)}=z_{\max }(h)} \\
\bar{z}(h) \square \frac{1}{L-1} \sum_{l=0, l \neq \hat{\delta}(h)}^{L-1} z_{l}(h) \\
\lambda(h) \square z_{\max }(h) / \bar{z}(h)
\end{gathered}
$$

Then the signal recognition rule can be given:

$$
\lambda(h) \underset{H_{0}}{\stackrel{H_{1}}{\gtrless}} \lambda_{0}
$$

where $\lambda(h)$ is the detection variable, $\lambda_{0}$ is the threshold which can be defined by the false alarm probablity. If the decision result is $H_{1}, \hat{\delta}(h)$ is the code acquisition position.

\section{SignAl RECONSTRUCTION}

We define the $\hat{\delta}(h)$ is the code acquisition position of the k-th path, which is the position of correlation peak $z_{\max }(h)$. Then the amplitude of k-th path $\alpha_{k}$ can be evaluated by

$$
\alpha_{k} \square \sqrt{z_{\max }(h) /\left(W \cdot L^{2}\right)}
$$

The k-th path signal $x_{k}(n)$ can be reconstructed according to the amplitude $\alpha_{k}$ and the code acquisition position $\delta_{k}(h)$,

$$
x_{k}(n)=\alpha_{k} \cdot \sum_{i=0}^{W \cdot L-1}\left[\tilde{c}(i) \cdot g\left(\left(n-i \cdot Q-\delta_{k}(h)\right) \frac{T_{c}}{Q}\right)\right]
$$

where $\mathrm{Q}$ is the over sampling factor, $\tilde{c}$ is the consecutive local PN code which the number is $\mathrm{W}, T_{c}$ is the chip period.

$$
g(t)=g_{T}(t) * g_{R}(t)
$$

where $g_{T}(t)$ is the sending filter and $g_{R}(t)$ is receiving filter,

\section{THE NOVEL SYNCHRONIZATION ALGORITHM BASED ON THE SIGNAL RECONSTRUCTION}

After signal reconstruction, the interference between multipath can be cancelled.

$$
\tilde{r}_{k+1}(i)=\tilde{r}_{k}(i)-x_{k}(i)
$$

Then the parameters of the next path can be evaluated more precisely.

The novel synchronization algorithm based on signal reconstruction contains six steps, as follows. 
Step 1: The receiving signal is filtered the receiving filter and then oversampled;

Step 2: The filtered signal is over sampled;

Step 3: The sampled signals are sent to identical matched filters. The filters form a transversal type correlator and output the correlation signal between the input signal and a reference spreading code;

Step 4: The signal recognition finds the correlation peak position and make a decision whether the signal is active. If this is true, the signal reconstruction is executed; if this is not true, go back to step 1 .

Step 5: The parameters of k-th path can be evaluated and the k-th path signal can be reconstructed.

Step 6: The interference between different paths can be cancelled before go back to step3.

The scheme of novel synchronization algorithm based on signal reconstruction is shown in the figure 2.

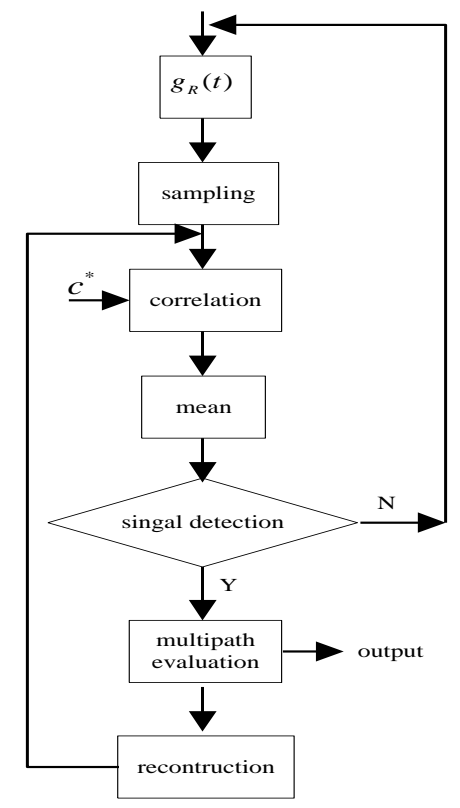

Figure 2. Scheme of the CSS-NT algorithm

\section{SiMUlation ResUlts}

In this section we present some simulation results to demonstrate the synchronization performance of the novel algorithm based on signal reconstruction (SA-SR). The simulation is made based on DS-SS/MPSK in HF channels. The simulation parameters are shown in the Table.1.

TABLE.1 PARAMETERS OF THE SIMULATION

\begin{tabular}{|c|c|}
\hline Parameter & value \\
\hline L & 63 \\
\hline Q & 4 \\
\hline W & 4 \\
\hline M & 4 \\
\hline multipath delay & 2 \\
\hline fading channel gains & 6 \\
\hline CAFR & {$[0.8 .0 .25]$} \\
\hline
\end{tabular}

In figure 3, the distribution of the correlation after step 3 is given. We can see that the first path is so strong and the second path cannot be found.

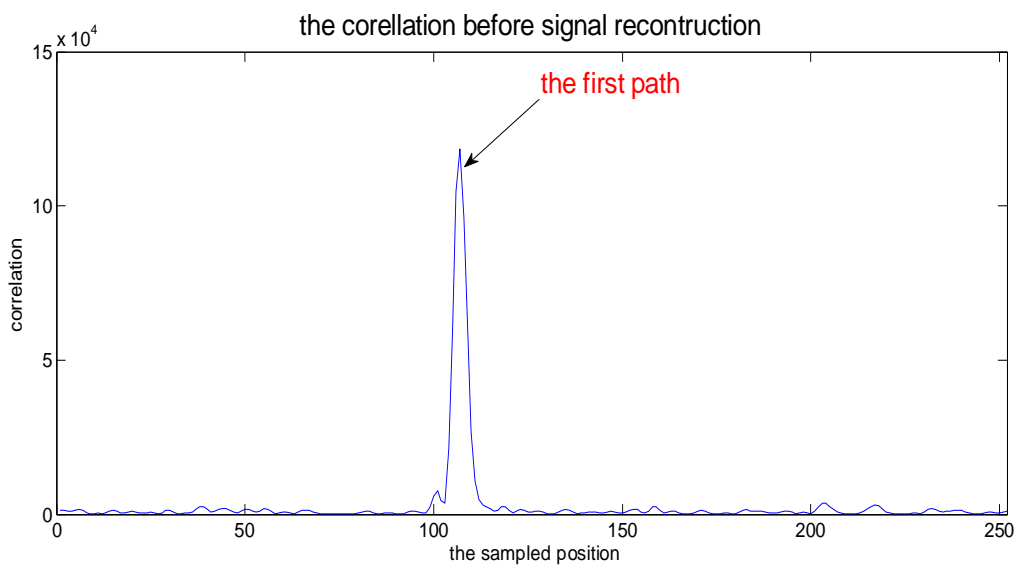

Figure 3. The distribution of the correlation before signal reconstruction

After signal reconstruction and the interference cancelling, the second path can be defined precisely, which is shown in figure 4. 
the correlation after signal recontruction

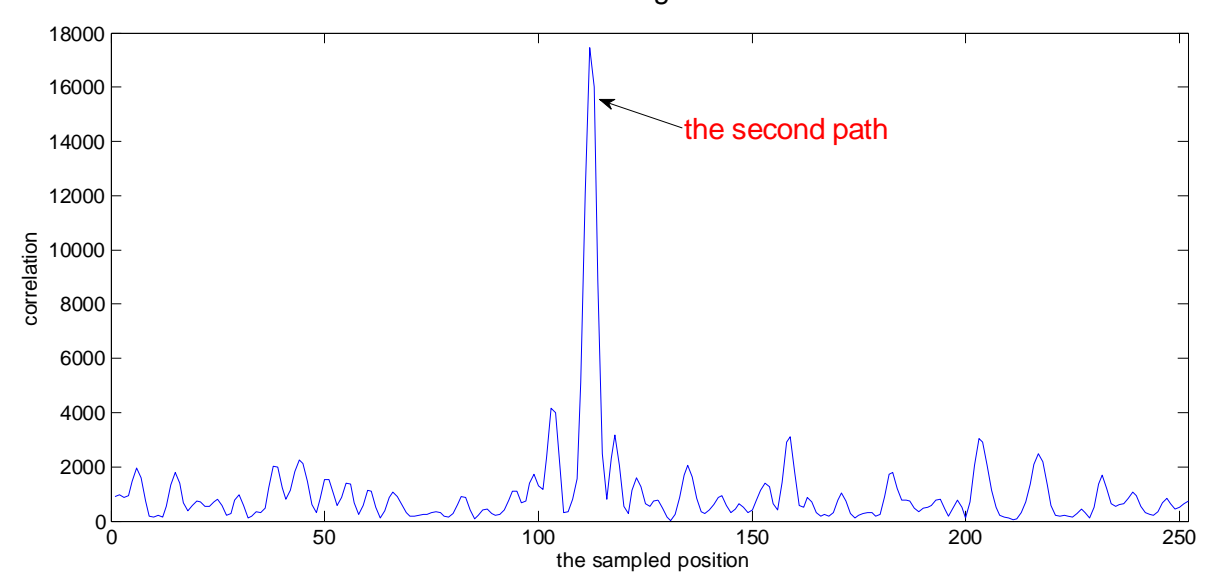

Figure 4. The distribution of the correlation after signal reconstruction

In figure 5 , the mean evaluated position deviation of multipath is given. We can see that the first path can be evaluated easily. When using the typical method, the second path cannot be found precisely before signal reconstruction. However, the novel algorithm can work well when the SNR is larger than $-2 \mathrm{~dB}$.

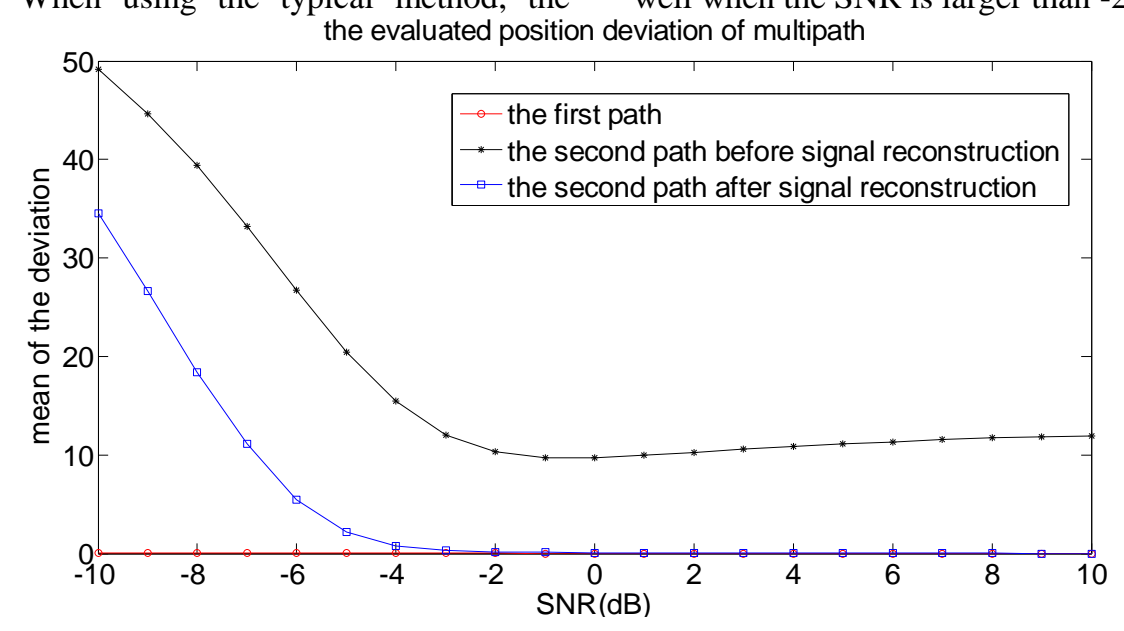

Figure 5. The mean evaluated position deviation of multipath

In figure 6 , the mean evaluated amplitude deviation of multipath is given. We can see that the first path can be evaluated easily. After using the signal reconstruction, the

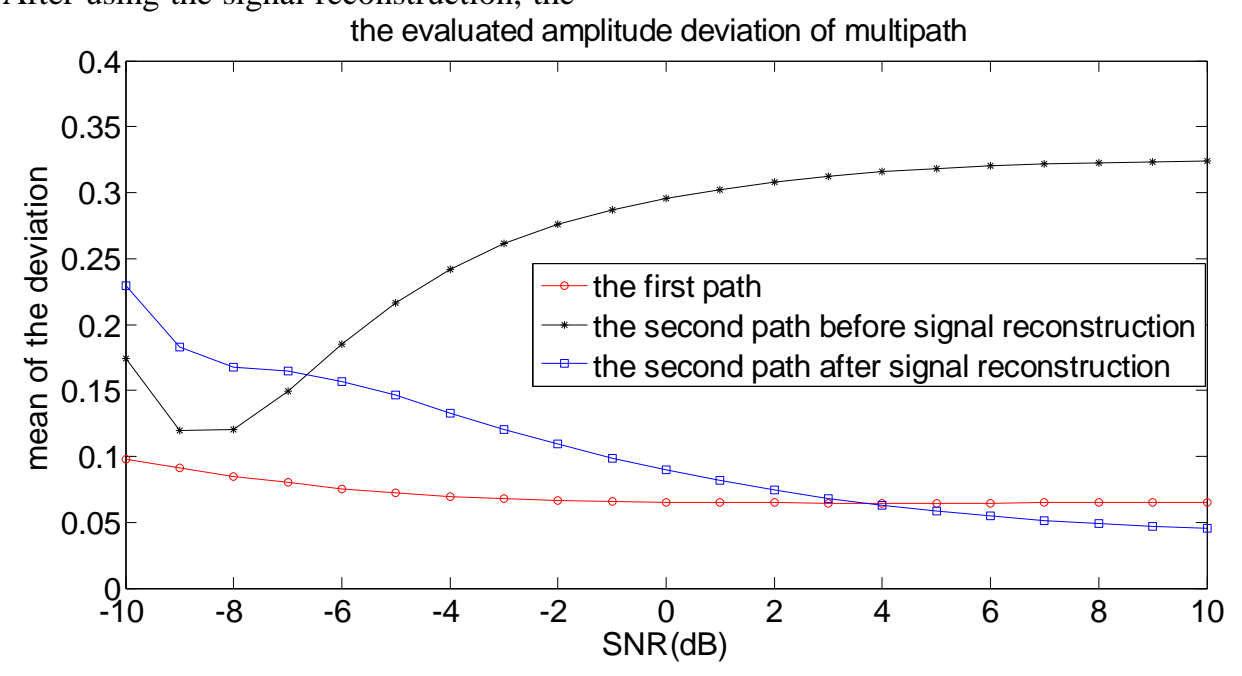

Figure 6. The mean evaluated amplitude deviation of multipath 
In figure 7 , the variance of evaluated multipath amplitude deviation is given. We can see that the amplitude evaluation performance of the novel algorithm is better than the typical method, which is the same with figure 6 .

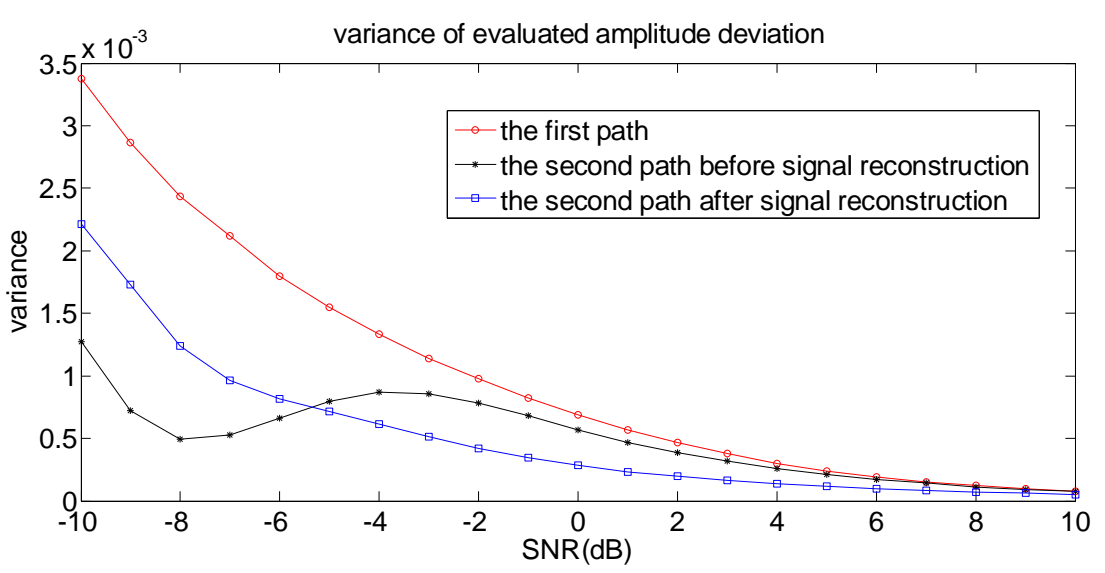

Figure 7. The variance of the evaluated multipath position deviation

In figure 8 , the detection probability is given when the false alarm probability is constant. We can see that when the first path is strong, it can be evaluated easily. The detection performance of the novel algorithm can be improved through using signal reconstruction which is better than the typical method.

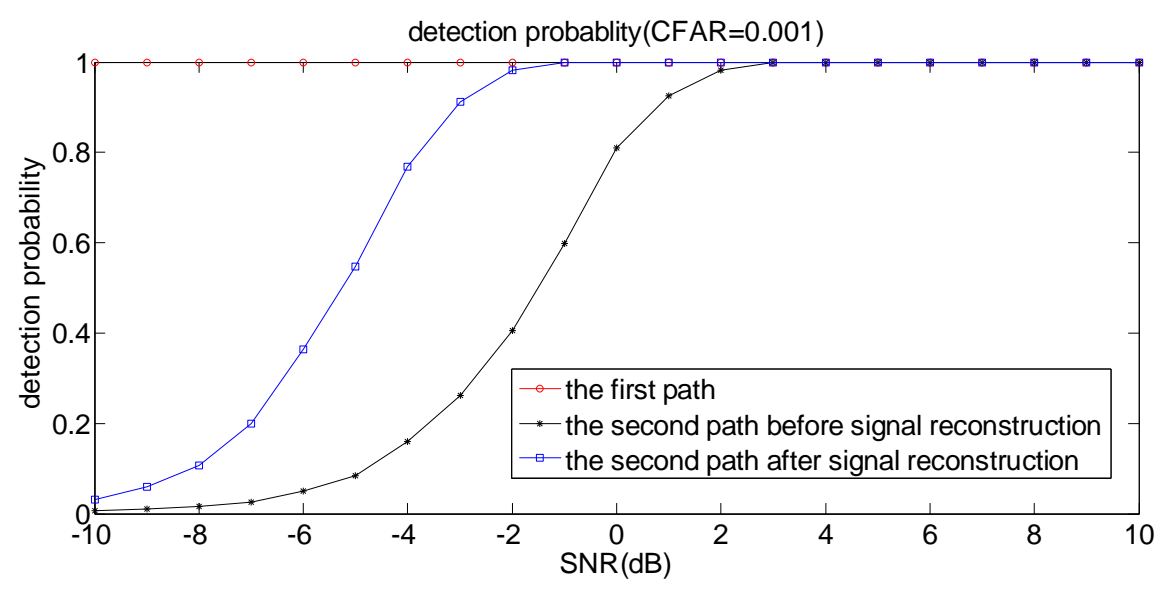

Figure 8 . The detection performance of the novel algorithm

It can be shown that, the novel synchronization algorithm based on signal reconstruction is better than the typical method which is shown in figure 1 . When the multipath cannot be separated easily, the interference between different paths can reduce the synchronization performance. When the signal reconstruction is used, the interference between different paths can be cancelled and the parameter evaluation can be done more precisely.

\section{CONCLUSION}

The DS-SS synchronization method in multipath HF channel is studied. A novel timing synchronization algorithm based on signal reconstruction is proposed in multipath fading HF channel. Through signal reconstructing and interference cancelling, the path position and multipath delay of different paths can be evaluated precisely. The simulation results show that the detection performance can be improved, and this new timing algorithm can easily separate different paths which cannot be done by traditional RAKE receiver. The algorithm design for multi-reporting users is our future research.

\section{ACKNOWLEDGEMENT}

This research was financially supported by the China Postdoctoral Science Foundation under Grant No 2014M552611.

\section{REFERENCES}

[1] G. R. Cooper and R. W. Nettleton, A spread spectrum technique for high capacity mobile communications, IEEE Trans. Veh. Technol, vol. 27(1978) 264-275

[2] R. L. Pickholtz, D. L. Schilling, and L. B. Milstein, Theory of spread spectrum communications-A tutorial, IEEE Trans. Commun, 30(1982) 855-884.

[3] R. L. Pickholtz, L. B. Milstein, and D. L. Schilling, Spread spectrum for mobile communications, IEEE Trans. Veh. Technol, 40(1991) 313-321.

[4] W. R. Braun, PN acquisition and tracking performance in DS/CDMA systems with symbol-length spreading sequences, IEEE Trans. Commun, 45(1997) 1595-1601.

[5] M. K. Simon, Noncoherent pseudonoise code tracking performance of spread spectrum receiver, IEEE Trans. Commun, 25(1977) 327-345.

[6] Youli Wu, Huili Fan, Synchronization acquisition algorithm for OFDM system, ICEIEC 2015,262-265. 
[7] Tao Chen, Yongfei Ding, Ruifan Pang, et, Code acquisition of a DS-CDMA receiver with antenna arrays and blind adaptation for air traffic control, DASC 2016, 1-6.

[8] Gang Wei, Fei Ji, Juan Fu, Modelling and Simulation of HF Communication Systems, Publishing House of Electronics Industry, 2007, 173-176.

[9] A.P.Clark, S.NAbdullah,S.F.Hau, Improved time synchronization for an $\mathrm{HF}$ radio modem, IEE Proceeding I-Communications, Speech and Vision,136(1989) 47-56.

[10] Yasuo Suzuki, Eisuke Kudoh, Member, IEEE, and Shigeaki Ogose, DS-CDMA RAKE Receiver with Time-Window Control Loop (TWCL) in Multipath Fading Environment. IEEE Tran. Vehicular Technology, 49(2000) 167-172.

[11] Latifa Hacini, Atef Farrouki, Zoheir Hammoudi, Hybird acquisition of PN codes using automatic multipath censoring in DS-CDMA communications, ACTEA 2009, 269-264. 